\title{
Bronchial Foreign Body Aspiration due to Polycarbophil Calcium
}

\author{
Takehiro Tsuchiya ${ }^{1}$, Atsushi Sano ${ }^{1}$, Mai Kaneko ${ }^{2}$, Satomi Mizutani ${ }^{2}$ and Tsutomu Fukuda ${ }^{2}$
}

\begin{abstract}
:
Polycarbophil calcium (Polyful ${ }^{\circledR}$; Mylan, Tokyo, Japan) is a stool stabilizer that absorbs liquid and swells to form a soft, bulky mass. A 75-year-old woman experienced sore throat and difficulty breathing immediately after taking the drug. Chest computed tomography showed a foreign body in the right intermediate bronchus. Bronchoscopy showed a white mass blocking the right intermediate bronchus. Since the mass was very fragile, we performed suctioning while breaking up the mass with a suction tube. The mass consisted of polycarbophil calcium. Since aspirated polycarbophil calcium swells and can obstruct bronchi, complete removal is crucial.
\end{abstract}

Key words: bronchial foreign body, bronchial aspiration, polycarbophil calcium, complication

(Intern Med Advance Publication)

(DOI: 10.2169/internalmedicine.7030-21)

\section{Introduction}

Polycarbophil calcium (Polyful ${ }^{\circledR}$; Mylan, Tokyo, Japan) is a stool stabilizer that absorbs liquid in the intestines and swells to form a soft, bulky mass. Thus far, aspiration of polycarbophil calcium has rarely been reported $(1,2)$.

We herein report a case of foreign body (FB) aspiration due to polycarbophil calcium. A decreased swallowing function due to Parkinson's disease was thought to be the cause of FB aspiration in this case.

\section{Case Report}

A 75-year-old woman took polycarbophil calcium regularly to treat irritable bowel syndrome. One day, she experienced sore throat and difficulty breathing immediately after taking the drug. There were no other symptoms. She was referred to our hospital the next day. We heard expiratory wheezing in her right middle lung field. The wheezing gradually became inaudible over a few hours. Chest radiography showed a nodule in the right hilum and a shift of the mediastinum to the left (Fig. 1A). At 72 years old, she left lower lobectomy due to lung metastasis of pancreatic carci- noma. The left shift of the mediastinum and decreased left thoracic volume were thus considered to be related to the prior operation.

Chest computed tomography (CT) showed a $\mathrm{FB}$ in the right intermediate bronchus (Fig. 1B). An arterial blood gas analysis indicated the following: $\mathrm{pH}$ 7.377, $\mathrm{pCO}_{2} 47.8$ $\mathrm{mmHg}$, and $\mathrm{pO}_{2} 59.8 \mathrm{mmHg}$ while breathing room air. Left lower lobectomy and FB aspiration were thus thought to have caused type 2 respiratory failure. Therefore, we administered oxygen and performed flexible bronchoscopy for examination and treatment.

Bronchoscopy showed a white mass blocking the intermediate bronchus (Fig. 2A, B). The mass was very fragile, and grasping forceps caused fragmentation (Fig. 3A). We therefore performed suctioning while breaking up the mass with a suction tube several dozen times. This cleared the bronchus. We observed erosion of the bronchial mucosa (Fig. 3B). The mass was considered to consist of polycarbophil calcium because she had experienced sore throat and difficulty breathing immediately after taking the drug.

After bronchoscopy, the patient's sore throat and difficulty breathing rapidly lessened. During bronchoscopy, we noted that her cough reflex was very weak. A subsequent examination showed that she had Parkinson's disease. Thus, the

${ }^{1}$ Department of Thoracic Surgery, Chigasaki Municipal Hospital, Japan and ${ }^{2}$ Department of Respiratory Medicine, Chigasaki Municipal Hospital, Japan

Received: January 6, 2021; Accepted: March 17, 2021; Advance Publication by J-STAGE: April 26, 2021

Correspondence to Dr. Takehiro Tsuchiya, tatsuchiya-ths@umin.ac.jp 


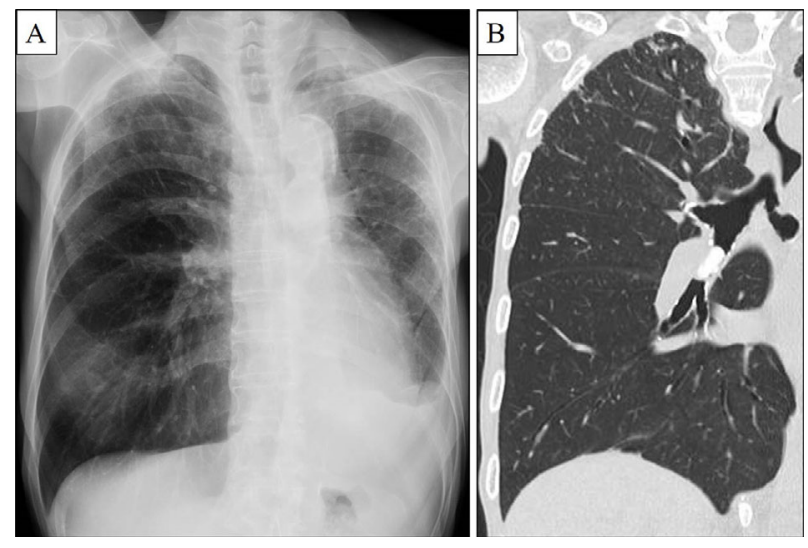

Figure 1. A: Chest radiography showed a nodule in the right hilum. In addition, left shift of the mediastinum and decreased left thoracic volume due to left lower lobectomy were observed. B: Chest computed tomography showed obstruction of the right intermediate bronchus. cause of FB aspiration was thought to be a decreased swallowing function.

\section{Discussion}

The symptoms and causes of aspiration are diverse. Patients may present with acute choking (3), or they may experience sore throat, recurrent pneumonia, mild dyspnea, or even no symptoms (4). Our patient experienced sore throat and difficulty breathing immediately after taking polycarbophil calcium. A decreased swallowing function due to Parkinson's disease was thought to be the cause of the FB aspiration. In addition, constipation might be associated with automatic dysfunction in Parkinson's disease. Therefore, when clinicians evaluate patients with FB aspiration, they should evaluate the swallowing function and identify the disorder causing the decreased swallowing function. Indeed, previous studies have shown that patients with a decreased
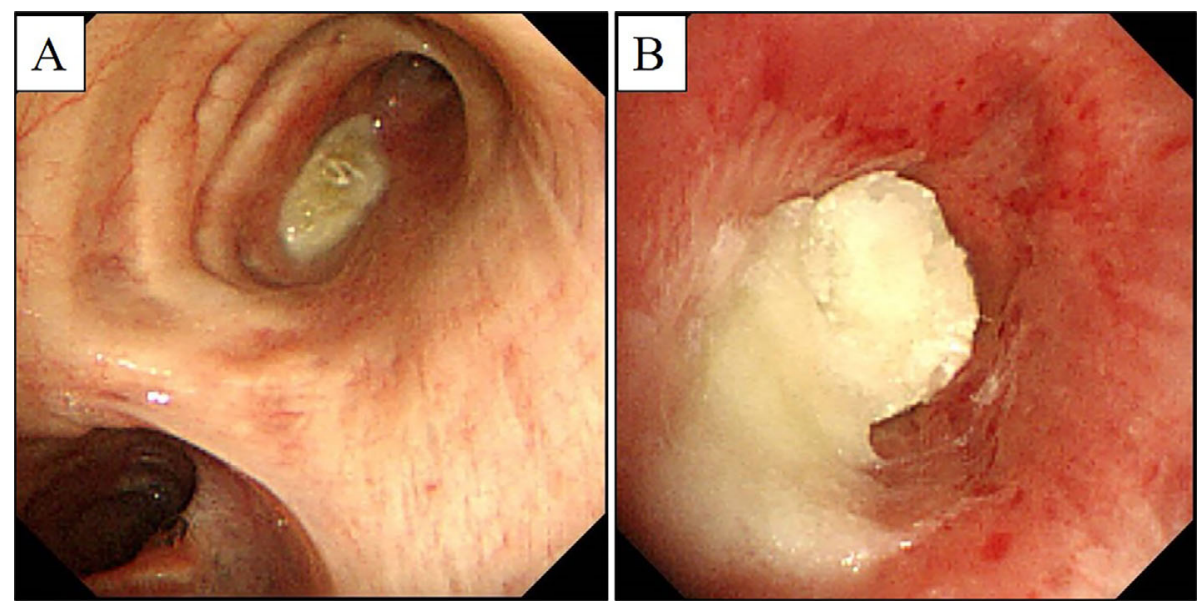

Figure 2. A: Bronchoscopy showed obstruction of the right intermediate bronchus due to a white material. B: Bronchoscopy showed the white material expanding and adhering to the bronchi.
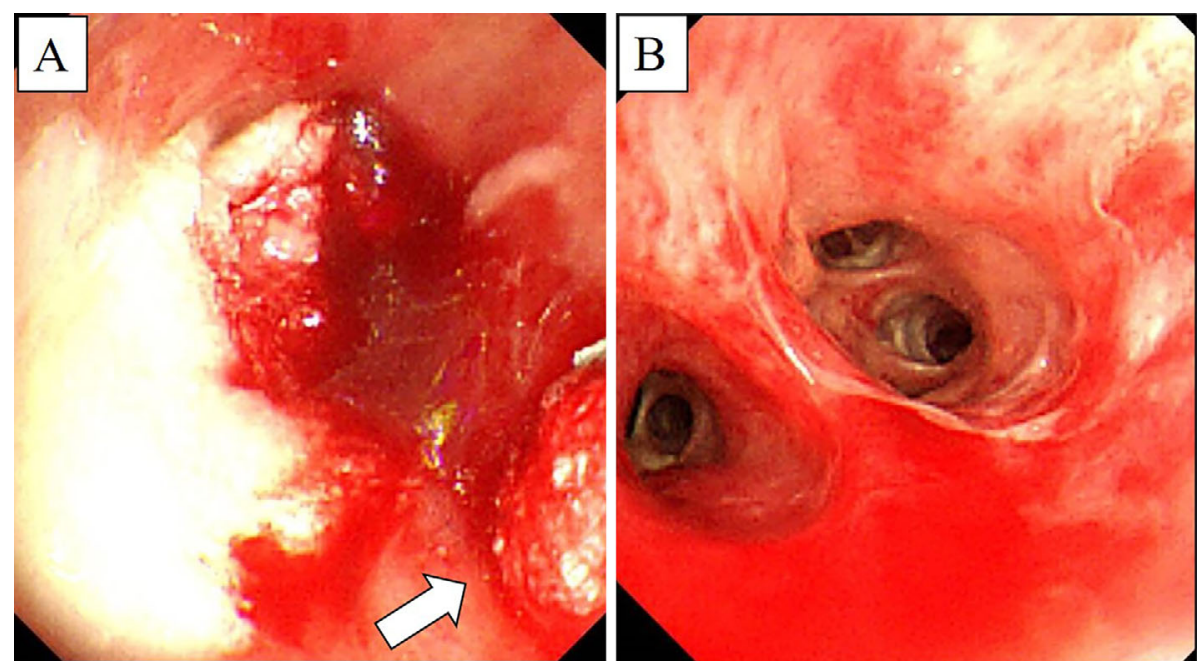

Figure 3. A: Bronchoscopy showed that the mass was very fragile. Grasping forceps caused fragmentation (arrowhead). B: Bronchoscopy showed patency of the right intermediate bronchus after suctioning through a fiberscope. 
swallowing function often have an underlying risk factor, such as neuromuscular disease, head trauma, alcohol intoxication, or altered sensorium (5-8). Another report showed that the composition of FBs included organic compounds in $25.6 \%$; metal, $41.0 \%$; teeth, $20.5 \%$; plastic, $10.3 \%$; and drug tablets, $2.6 \%$ (9). Thus, drug tablet FBs are rare, as are reports of polycarbophil calcium FBs $(1,2)$. Polycarbophil calcium is used as a stool stabilizer. Bulk-forming laxatives absorb liquid in the intestines and swell to form a soft, bulky mass. The bulky mass stimulates the intestinal muscles, speeding up stool transit time through the colon. Polycarbophil calcium absorbs approximately 10 times its own weight in water under acidic conditions. However, the degree of swelling markedly increases above $\mathrm{pH} 4.0$, reaching 70 times its weight at neutral $\mathrm{pH}(9)$. In the present case, CT showed that the volume of polycarbophil calcium had increased from $17.8 \times 7.6 \times 5.9 \mathrm{~mm}$ before ingestion to $17.9 \times$ $12.0 \times 9.6 \mathrm{~mm}$ after ingestion. The volume increased 2.58 times over the course of one day. As a result, polycarbophil calcium blocked the right intermediate bronchus. FBs can induce numerous complications, such as granulation tissue formation, recurrent pneumonia, atelectasis, and endobronchial stenosis and scarring (10). Therefore, a delayed diagnosis and removal can cause serious problems. Even if a patient does not have a history of aspiration, the differential diagnosis of cough or dyspnea should include FB aspiration, which will facilitate the early detection and treatment. The equipment used for FB removal depends on the shape and hardness of the material. Grasping or tooth (alligator) forceps are used to remove flat, thin inorganic, or hard organic FBs (11). Whereas a suction tube, fish net, or Dormia basket is used to remove soft FBs, as grasping forceps can cause fragmentation (9). In this case, the polycarbophil calcium FB became very fragile with expansion, and grasping forceps caused fragmentation. We therefore suctioned the material while breaking it apart with a suction tube. In each case of polycarbophil calcium aspiration reported to date, suction successfully resolved the problem $(1,2)$.

In conclusion, polycarbophil calcium aspiration can obstruct bronchi. This diagnosis should be kept in mind when evaluating patients who take polycarbophil calcium orally. In addition, since residual polycarbophil calcium can reobstruct bronchi and prevent bronchial expansion, complete removal is crucial.

The authors state that they have no Conflict of Interest (COI).

\section{Funding}

The author(s) received no financial support for the research, authorship, and/or publication of this article.

\section{References}

1. Enokido T, Shibuya H, Osamura K, Ohtani M. Polycarbophil Calcium Loged in the Bronchus. Intern Med 56: 2075-2076, 2017.

2. Kobayashi N, Itoh H, Matsui $\mathrm{K}$, et al. A case of foreign body aspiration with polycarbophil calcium. J Jpn Soc Bronchol 38: 242, 2016 (in Japanese).

3. Sano A, Fukuda T. Acute respiratory failure due to bronchial obstruction by mochi. J Bronchol Intervent Pulmonol 27: 46-48, 2017.

4. Jeffrey $\mathrm{Ng}$, Kim S, Chang B, et al. Clinical features and treatment outcomes of airway foreign body aspiration in adults. J Thorac Dis 11: 1056-1064, 2019.

5. Limper AH, Prakash UB. Tracheobronchial foreign bodies in adults. Ann Intern Med 112: 604-609, 1990.

6. Cunanan OS. The flexible fiberoptic bronchoscope in foreign body removal. Experience in 300 cases. Chest 73: 725-726, 1978.

7. Mise K, Jurcev Savicevic A, Pavlov N, et al. Removal of tracheobronchial foreign bodies in adults using flexible bronchoscopy: experience 1995-2006. Surg Endosc 23: 1360-1364, 2009.

8. Boyd M, Chatterjee A, Chiles C, et al. Tracheobronchial foreign body aspiration in adults. South Med J 102: 171-174, 2009.

9. Sehgal IS, Dhooria S, Ram B, et al. Foreign Body Inhalation in the Adult Population: Experience of 25,998 Bronchoscopies and Systematic Review of the Literature. Respir Care 60: 1438-1448, 2015.

10. Yamada T, Nagata O, Tamai I, et al. Physicochemical Properties of Calcium Polycarbophil, a Water-absorbing Polymer. 48: 665668, 1996.

11. Rafanan AL, Mehta AC. Adult airway foreign body removal. What's new? Clin Chest Med 22: 319-330, 2001.

The Internal Medicine is an Open Access journal distributed under the Creative Commons Attribution-NonCommercial-NoDerivatives 4.0 International License. To view the details of this license, please visit (https://creativecommons.org/licenses/ by-nc-nd/4.0/).

(C) The Japanese Society of Internal Medicine Intern Med Advance Publication 